\title{
Convex functions on Carnot groups
}

Petri Juutinen, Guozhen Lu, Juan J. Manfredi, Bianca Stroffolini

\begin{abstract}
We consider the definition and regularity properties of convex functions in Carnot groups. We show that various notions of convexity in the subelliptic setting that have appeared in the literature are equivalent. Our point of view is based on thinking of convex functions as subsolutions of homogeneous elliptic equations.
\end{abstract}

\section{Introduction}

In this article we consider convex functions on general Carnot groups. We consider several definitions, prove their equivalence, and establish basic regularity properties. The simpler case of the Heisenberg group was treated in [13].

A key observation is the realization that convexity depends only on the horizontal distribution and not on the particular base chosen to represent it (see section $\S 3$ below.) This allows us to use potential theoretic representation formulas developed by Bonfiglioli and Lanconelli [4] to approximate convex functions by smooth convex functions. Another new ingredient is Wang's extension [17] to Carnot groups of Bieske's uniqueness result for $\infty$-harmonic functions in the Heisenberg group.

Let us briefly recall some basic facts about Carnot groups. A Carnot group $\mathcal{G}$ of step $r \geq 1$ is a simply connected nilpotent Lie group whose Lie algebra $\mathfrak{g}$ is stratified. This means that $\mathfrak{g}$ admits a decomposition as a vector space sum

$$
\mathfrak{g}=\mathfrak{g}_{1} \oplus \mathfrak{g}_{2} \oplus \cdots \oplus \mathfrak{g}_{r}
$$

such that

$$
\left[\mathfrak{g}_{1}, \mathfrak{g}_{j}\right]=\mathfrak{g}_{j+1}
$$

for $j=1, \ldots, r$ with $\mathfrak{g}_{k}=\{0\}$ for $k>r$. Note that $\mathfrak{g}$ is generated as a Lie algebra by $\mathfrak{g}_{1}$.

2000 Mathematics Subject Classification: Primary: 49L25, 35J70. Second.: 35J67, 22E30. Keywords: Horizontal convexity, viscosity convexity, Carnot groups, subelliptic equations. 
Let $m_{j}=\operatorname{dim}\left(\mathfrak{g}_{j}\right)$ and choose a basis of $\mathfrak{g}_{j}$ formed by left-invariant vector fields

$$
\mathfrak{X}=\left\{X_{i, j}: i=1, \ldots, m_{j}, j=1, \ldots, r\right\} .
$$

The dimension of $\mathcal{G}$ as a manifold is $n=m_{1}+\cdots+m_{r}$. The horizontal tangent space at a point $p \in \mathcal{G}$ is the $m_{1}$-dimensional subspace of $\mathfrak{g}$ spanned by $\left\{X_{1,1}(p), \ldots, X_{1, m_{1}}(p)\right\}$. From now on we will drop the first index and indicate by $\left\{X_{1}, \ldots, X_{m_{1}}\right\}$ a frame of vector fields that span the first layer $\mathfrak{g}_{1}$. With the above notations the horizontal subspace can be identified with the left translation by $p$ of $\mathcal{G}_{1}=\exp \left(\mathfrak{g}_{1}\right)$, the horizontal subspace at the origin, via the exponential mapping; that is, we have

$$
p \cdot \mathcal{G}_{1}=\text { linear-span }\left\{X_{1}(p), \ldots, X_{m_{1}}(p)\right\} .
$$

A horizontal curve $\gamma(t)$ is a piece-wise smooth curve whose tangent vector $\gamma^{\prime}(t)$ is in the horizontal tangent space $\gamma(t) \cdot \mathcal{G}_{1}$ whenever it exists. Given two points $p$ and $q$ we consider the set of all possible horizontal curves joining these points:

$$
\Gamma(p, q)=\{\gamma \text { horizontal curve }: \gamma(0)=p, \gamma(1)=q\}
$$

This set is never empty by Chow's theorem (see for example [5]). For convenience, we fix an ambient Riemannian metric in $\mathfrak{g}$ so that $\mathfrak{X}$ is an orthonormal frame and the Riemannian volume element agrees with the Haar measure of $\mathcal{G}$, and this Haar measure is the Lebesgue measure in $\mathbb{R}^{n}$. This is always possible, see [9, Chapter 1].

The Carnot-Carathéodory distance is then defined as the infimum of the length of horizontal curves of the set $\Gamma$ :

$$
d_{C C}(p, q)=\inf _{\Gamma(p, q)} \int_{0}^{1}\left|\gamma^{\prime}(t)\right| d t
$$

It depends only of the restriction of the ambient Riemannian metric to the horizontal distribution generated by the horizontal tangent spaces. The Carnot-Carathéodory ball of radius $R$ centered at a point $p$ is given by

$$
B(p, R)=\left\{q \in G: d_{C C}(p, q)<r\right\} .
$$

Using the so-called ball-box theorem ([5]), the volume of a ball can be easily estimated to be

$$
\operatorname{vol}(B(0, R)) \sim r^{Q}
$$

where $Q=\sum_{j=1}^{m_{j}} j m_{j}$ is the homogeneous dimension of $\mathcal{G}$. 
The natural (non-isotropic) dilations $\delta_{t}$ in $\mathcal{G}$ are given in exponential coordinates by

$$
\delta_{t}\left(\exp \left(\sum_{j=1}^{r} \sum_{i=1}^{m_{j}} p_{i, j} X_{i, j}\right)\right)=\exp \left(\sum_{j=1}^{r} \sum_{i=1}^{m_{j}} t^{j} p_{i, j} X_{i, j}\right),
$$

for $t>0$. These dilations are group homomorphisms. For negative $t>0$ we define $\delta_{t}=\left(\delta_{-t}\right)^{-1}$.

For a smooth function $u: \mathcal{G} \mapsto \mathbb{R}$ the gradient can be written relative to $\mathfrak{X}$ as the vector

$$
D_{\mathfrak{X}} u=\left(X_{i, j} u\right)_{j=1 \ldots r, i=1, \ldots m_{j}} .
$$

The horizontal gradient of $u$ at a point $p$ is then the projection of the gradient of $u$ at $p$ onto the horizontal subspace $p \cdot \mathcal{G}_{1}$, and is given by

$$
D_{\mathrm{h}} u=\left(X_{1} u, \ldots, X_{m_{1}} u\right) .
$$

The symmetrized horizontal second derivative matrix, denoted by $\left(D_{\mathrm{h}}^{2} u\right)^{*}$, is the $m_{1} \times m_{1}$ matrix with entries

$$
\left(D_{\mathrm{h}}^{2} u\right)_{i j}^{*}=\frac{1}{2}\left(X_{i} X_{j} u+X_{j} X_{i} u\right)
$$

for $i, j=1,2, \ldots, m_{1}$.

Let us recall the definition of convexity in the viscosity sense:

Definition 1.1. Let $\Omega \subset \mathcal{G}$ be an open set and $u: \Omega \rightarrow \mathbb{R}$ be an uppersemicontinuous function. We say that $u$ is convex in $\Omega$ if

$$
\left(D_{\mathrm{h}}^{2} u\right)^{*} \geq 0
$$

in the viscosity sense. That is, if $p \in \Omega$ and $\phi \in C^{2}$ touches $u$ from above at $p(\phi(p)=u(p)$ and $\phi(q) \geq u(q)$ for $q$ near $p)$ we have $\left(D_{\mathrm{h}}^{2} \phi\right)^{*}(p) \geq 0$.

This definition is compatible with the stratified group structure since convexity is preserved by left-translations and by dilations. As in the case of the Heisenberg group, uniform limits of convex functions are convex and the supremum of a family of convex functions is convex, since these results hold for viscosity subsolutions in general.

Notice that we require the a-priori regularity assumption of upper-semicontinuity as it is done in the definition of sub-harmonic functions. This is not needed when horizontally convex functions are considered. These are defined by requiring that whenever $p \in \Omega$ and the horizontal segment $h \in \mathcal{G}_{1}$ are such that the segment $\left[p \cdot h^{-1}, p \cdot h\right] \subset \Omega$ the function of one real variable

$$
t \mapsto u\left(p \cdot \delta_{t}(h)\right)
$$

is convex for $-1<t<1$. 
It was established in [13] that upper-semicontinuous horizontally convex functions are indeed convex in the sense of Definition 1.1. In this paper we will show that the reciprocal is also true (see Theorem 3.1 below.) This equivalence has also been established independently by Wang [18] and Magnani [14].

In the Heisenberg group Balogh and Rickley [5] proved that condition (1.1) by itself, without requiring upper-semicontinuity, suffices to guarantee that $u$ is continuous - and therefore Lipschitz continuous- and also showed that horizontally convex functions are convex in the sense of Definition 1.1.

While this manuscript was in preparation we learned about the monotonicity and comparison results of Gutiérrez and Montanari [10, 11] in groups of Heisenberg type. It follows from these results that a convex function is in the space $B V_{h \text {, loc }}^{2}$ of functions whose second derivatives (in the homogeneous sense) are measures. A theorem of Ambrosio and Magnani [1] implies then the twice pointwise differentiability a.e. of convex functions. Theorems of Aleksandrov type for Carnot groups of step 2 can also be found in [8] and [14].

We would like to thank Giovanna Citti for pointing out an error in a previous version of this manuscript.

\section{Convexity in the Viscosity Sense in $\mathbb{R}^{n}$}

In order to illustrate our approach in the case of general Carnot groups, we present here the Euclidean version of Theorem 3.1 below. Let $\Omega$ be a domain in $\mathbb{R}^{n}$. Consider a continuous function $F(x, z, p, M)$ in $\Omega \times \mathbb{R} \times \mathbb{R}^{n} \times \mathcal{S}^{n}$ that satisfies

$$
\left\{\begin{array}{l}
F(x, z, p, 0)=0 \\
F(x, z, p, M) \leq F\left(x, z^{\prime}, p, M\right) \text { if } z \leq z^{\prime}, \text { and } \\
F(x, z, p, M) \leq F\left(x, z, p, M^{\prime}\right) \text { if } M^{\prime} \leq M
\end{array}\right.
$$

The last two conditions indicate that $F$ is proper and degenerate elliptic according to the terminology of [6]. Here $\mathcal{S}^{n}$ denotes the class of $n \times n$ real symmetric matrices.

Theorem 2.1. Let $\Omega \subset \mathbb{R}^{n}$ be an open set and $u: \Omega \rightarrow \mathbb{R}$ be an uppersemicontinuous function. The following statements are equivalent:

i) whenever $x, y \in \Omega$ and the segment joining $x$ and $y$ is also in $\Omega$ we have

$$
u(\lambda x+(1-\lambda) y) \leq \lambda u(x)+(1-\lambda) u(y) \quad \text { for all } 0 \leq \lambda \leq 1
$$

ii) $u$ is a viscosity subsolution of all equations

$$
F\left(x, u(x), D u(x), D^{2} u(x)\right)=0,
$$


where $F(x, z, p, M)$ is a continuous function in $\Omega \times \mathbb{R} \times \mathbb{R}^{n} \times \mathcal{S}^{n}$ satisfying (2.1).

iii) $u$ is a viscosity subsolution of all linear equations with constant coeffcients

$$
F\left(x, u, D u, D^{2} u\right)=-\operatorname{trace}\left(A \cdot D^{2} u\right)=0,
$$

where $A \in \mathcal{S}^{n}$ is positive definite.

iv) $x \rightarrow u(A x)$ is subharmonic for all $A \in \mathcal{S}^{n}$ positive definite;

v) u satisfies the inequality

$$
D^{2} u \geq 0
$$

in the viscosity sense;

vi) $u$ satisfies $-\operatorname{trace}\left(A \cdot D^{2} u\right) \leq 0$ in the sense of distributions for all $A \in \mathcal{S}^{n}$ positive definite.

A function $u$ is convex if one of the above equivalent statements holds.

The equivalence between i), ii), iii), and v) was established in [13]. The two new conditions added in this theorem are iv) and vi).

Proof. The equivalence between vi) and iii) for any given matrix $A$ is part of viscosity folklore. This is just the simplest case of the theory of Hessian measures of Trudinger and Wang [15]. To prove the equivalence between iv) and iii) observe that $x \rightarrow u(A x)$ is subharmonic for all $A>0$ if and only if $\operatorname{trace}\left(A \cdot D^{2} u(x) A^{t}\right) \geq 0$ in the sense of distributions for all $A>0$. This occurs precisely when trace $\left(A^{t} \cdot A \cdot D^{2} u(x)\right) \geq 0$ in the sense of distributions for all $A>0$. Since every positive definite matrix $B$ has a positive definite square root $B=A^{2}=A^{t} \cdot A$, we see that $x \rightarrow u(A x)$ is subharmonic for all $A>0$ if and only if $\operatorname{trace}\left(B \cdot D^{2} u(x)\right) \geq 0$ in the sense of distributions for all $B>0$.

\section{Convexity in Carnot Groups}

A key observation is that the notion of convexity depends only on the horizontal distribution and not on the particular choice of a basis of $\mathfrak{g}_{1}$. More precisely, let us consider two linearly independent horizontal frames

$$
\mathfrak{X}_{\mathrm{h}}=\left\{X_{1}, \ldots, X_{m_{1}}\right\}, \quad \mathfrak{Y}_{\mathrm{h}}=\left\{Y_{1}, \ldots, Y_{m_{1}}\right\}
$$

and write $X_{i}=\sum_{j=1}^{m_{1}} a_{i j} Y_{j}$, for some constants $a_{i j}$. Let $A$ be the matrix with entries $a_{i j}$. The matrix $A$ is not singular and the following formula holds for any smooth function $\phi$

$$
\left(D_{\mathrm{h}, \mathfrak{x}}^{2} \phi(p)\right)^{*}=A\left(D_{\mathrm{h}, \mathfrak{Y}}^{2} \phi(p)\right)^{*} A^{t} .
$$

Thus the matrix $\left(D_{\mathrm{h}, \mathfrak{x}}^{2} \phi(p)\right)^{*}$ is positive definite if and only if $\left(D_{\mathrm{h}, \mathfrak{Y}}^{2} \phi(p)\right)^{*}$ is positive definite. 
Given a frame $\mathfrak{X}$ we denote by

$$
\Delta_{\mathfrak{X}} u=\sum_{i=1}^{m_{1}} X_{i}^{2} u
$$

the corresponding Hörmander-Kohn Laplacian.

The main result of this section is the analogue to Theorem 2.1. Consider continuous functions

$$
F: \mathcal{G} \times \mathbb{R} \times \mathbb{R}^{m_{1}} \times \mathcal{S}^{m_{1}} \rightarrow \mathbb{R}
$$

that are homogeneous, proper, and degenerate elliptic:

$$
\left\{\begin{array}{l}
F(p, z, h, 0)=0 \\
F(p, z, h, M) \leq F\left(p, z^{\prime}, h, M\right) \text { if } z \leq z^{\prime}, \text { and } \\
F(p, z, h, M) \leq F\left(p, z, h, M^{\prime}\right) \text { if } M^{\prime} \leq M
\end{array}\right.
$$

Theorem 3.1. Let $\Omega \subset \mathcal{G}$ be an open set and $u: \Omega \rightarrow \mathbb{R}$ be an uppersemicontinuous function. The following statements are equivalent:

i) whenever $p \in \Omega$ and $h \in \mathcal{G}_{1}$ are such that $\left[p \cdot h^{-1}, p \cdot h\right] \subset \Omega$ we have that the function of one real variable

$$
t \mapsto u\left(p \cdot \delta_{t}(h)\right)
$$

is convex for $-1<t<1$.

ii) $u$ is a viscosity subsolution of all equations

$$
F\left(p, u(p), D_{\mathrm{h}} u(p),\left(D_{\mathrm{h}}^{2} u(p)\right)^{*}\right)=0,
$$

where $F(x, z, p, M)$ satisfies (3.1).

iii) $u$ is a viscosity subsolution of all linear equations with constant coefficients

$$
F\left(p, u, D_{\mathrm{h}} u,\left(D_{\mathrm{h}}^{2} u\right)^{*}\right)=-\operatorname{trace}\left(A \cdot\left(D_{\mathrm{h}}^{2} u\right)^{*}\right)=0,
$$

where $A \in \mathcal{S}^{m_{1}}$ is positive definite.

iv) $u$ satisfies the inequality $-\Delta_{\mathfrak{Y}} u \leq 0$ in the viscosity sense for all frames $\mathfrak{Y}$ such that $\mathfrak{Y}_{\mathrm{h}}=A \mathfrak{X}_{\mathrm{h}}$, where $A \in \mathcal{S}^{m_{1}}$ is positive definite.

v) u satisfies the inequality

$$
\left(D_{\mathrm{h}}^{2} u\right)^{*} \geq 0
$$

in the viscosity sense.

vi) $u$ satisfies $-\operatorname{trace}\left(A \cdot\left(D_{\mathrm{h}}^{2} u\right)^{*}\right) \leq 0$ in the sense of distributions for all $A \in \mathcal{S}^{m_{1}}$ positive definite. 
A few remarks are in order. Condition i) is called horizontal convexity in [13] and $H$-convexity in [7]. Note that iv) is indeed the analogue of iv) in Theorem 2.1. Condition v) is called v-convexity in [13] and in [18].

The equivalence of the four viscosity related conditions ii), iii), iv) and v) follows easily from elementary linear algebra facts as in Theorem 2.1. Moreover if one of these conditions holds, then $u$ is locally bounded. This is the case because $u$ is always a subsolution of the corresponding $\infty$-Laplacian (see (4.1) below). The details in the case of the Heisenberg group are contained in the proof of Lemma 3.1 in [13].

To show that iv) implies vi) we may do it one matrix $A \in \mathcal{S}^{m_{1}}$ at a time. Thus, we may assume that $A$ is the identity matrix. If $u$ is a bounded viscosity subsolution of the Hörmander-Kohn Laplacian, it follows using the same proofs as in Lemma 2.2 and Lemma 2.3 from [13] that $u$ is weaksubsolution with first horizontal derivatives locally square integrable. If $u$ is not bounded below, we use the truncation $u_{M}(x)=\max \{-M, u(x)\}$ and standard limit theorems (see [16].)

To prove the equivalence of (i) with the other conditions we need to establish that convex functions can be approximated by smooth convex functions. Note that this is relatively easy to do for horizontally convex functions since the inequality (1.1) is preserved by convolution with a smooth mollifier (see the proof of Theorem 4.2 in [13].) Fortunately for us, Bonfiglioli and Lanconelli [4] have characterized subharmonic functions by a sub-mean value property and proved that subharmonic functions can be approximated by smooth subharmonic functions.

The following is a restatement of Lemma 4.2 in [4] adapted to our needs:

Lemma 3.1. Let $J \in C_{0}^{\infty}(\mathcal{G})$ be a nonnegative function supported in the unit ball $B(0,1)$ satisfying the condition $\int_{\mathcal{G}} J(p) d p=1$. For $\epsilon>0$ set $J_{\epsilon}(p)=\epsilon^{-Q} J\left(\delta_{1 / \epsilon}(p)\right)$. Suppose that $\Omega \subset \mathcal{G}$ is a domain and that $u \in L_{\mathrm{loc}}^{1}(\Omega)$ is an upper semicontinuous function. For $p \in \Omega_{\epsilon}=\{q \in \mathcal{G}: B(q, \epsilon) \subset \Omega\}$ define

$$
u_{\epsilon}(p)=\int_{\mathcal{G}} u(q) J_{\epsilon}\left(p \cdot q^{-1}\right) d q .
$$

Then if $u$ is subharmonic in $\Omega$ we have that $u_{\epsilon}$ is subharmonic in $\Omega_{\epsilon}$ and $u_{\epsilon} \epsilon$ $C^{\infty}\left(\Omega_{\epsilon}\right)$. Here subharmonic means $-\Delta_{\mathfrak{X}} u \leq 0$ in the sense of distributions.

An immediate consequence of this lemma is that vi) implies iv) since the implication holds for smooth functions, and viscosity subsolutions are preserved by locally uniform limits. The important feature of this lemma for our purposes is that the definition of $u_{\epsilon}$ is independent of the frame.

Lemma 3.2. Convex functions are locally uniform limits of smooth convex functions. 
Proof. Let $u$ be convex. Then $u$ is subharmonic relative to all frames $\mathfrak{Y}$ such that $\mathfrak{Y}_{\mathrm{h}}=A \mathfrak{X}_{\mathrm{h}}$, where $A \in \mathbb{S}^{m_{1}}$ is positive definite. By the previous lemma the smooth function $u_{\epsilon}$ is also subharmonic relative to all frames $\mathfrak{Y}$. Therefore, $u_{\epsilon}$ is convex.

We can now conclude the proof of Theorem 3.1 by quoting verbatim the proofs of Lemma 4.2 and Proposition 4.1 in [13].

We recall that a typical example of a convex function in the Heisenberg group $\mathcal{H}$ that is not convex in the Euclidean sense is given by

$$
v(x, y, z)=x^{2}+y^{2}-\varepsilon z^{2}
$$

in a neighborhood of the point $(0,0,0)$.

\section{Regularity of Convex Functions}

In this section we have the analogue of Theorem 3.1 in [13] for general Carnot groups. The point of view adapted in [13] is to use the fact that convex functions are subsolutions of all homogeneous elliptic equations. In particular we consider the Hörmander-Kohn Laplace equation

$$
-\Delta_{\mathrm{h}} u=-\left(X_{1}^{2} u+\cdots+X_{m}^{2} u\right)=0
$$

and the subelliptic $\infty$-Laplace equation

$$
-\Delta_{\infty, \mathrm{h}} u=-\sum_{i, j=1}^{m}\left(X_{i} u\right)\left(X_{j} u\right)\left(X_{i} X_{j} u\right)=0 .
$$

These equations can certainly be written in the form

$$
F\left(p, u(p), D_{\mathrm{h}} u(p),\left(D_{\mathrm{h}}^{2} u\right)^{*}(p)\right)=0
$$

for a continuous function $F$ satisfying (3.1).

Subsolutions of (4.1) are Lipschitz continuous. This was established by Jensen [12] in the Euclidean case and in [13] in the subelliptic case. This immediately gives a Lipschitz bound for convex functions.

The other ingredient used in the proof of Theorem 3.1 in [13] was Bieske's extension to the Heisenberg group of Jensen's uniqueness theorem for $\infty$-harmonic functions ([12], [3].) Recently Wang [17] has extended this theorem to general case of Carnot groups. The proof of Theorem 3.1 in [13] can now be repeated verbatim to get:

Theorem 4.1. Let $\Omega \subset \mathcal{G}$ be an open set and $u: \Omega \rightarrow \mathbb{R}$ be a convex function. Let $B_{R}$ be a ball such that $B_{4 R} \subset \Omega$. Then $u$ is locally bounded and we have

$$
\|u\|_{L^{\infty}\left(B_{R}\right)} \leq C f_{B_{4 R}}|u| d x
$$


Moreover, $u$ is locally Lipschitz and we have the bound

$$
\left\|D_{\mathrm{h}} u\right\|_{L^{\infty}\left(B_{R}\right)} \leq \frac{C}{R}\|u\|_{L^{\infty}\left(B_{2 R}\right)} .
$$

Here $C$ is a constant independent of $u$ and $R$. If, in addition, $u$ is $C^{2}$, then the symmetrized horizontal second derivatives are nonnegative

$$
\left(D_{\mathrm{h}}^{2} u\right)^{*} \geq 0
$$

\section{References}

[1] Ambrosio, L. And Magnani, V.: Weak differentiability of BV functions on stratified groups. Math. Z. 245 (2003), no. 1, 123-153.

[2] BAlOGH, Z. AND Rickly, M.: Regularity of convex functions on Heisenberg groups. Ann. Sc. Norm. Super. Pisa Cl. Sci (5) 2 (2003), 847-868.

[3] Bieske, T.: On $\infty$-harmonic functions on the Heisenberg group. Comm. Partial Differential Equations 27 (2002), no. 3-4, 727-761.

[4] Bonfiglioli, A. and Lanconelli, E.: Subharmonic functions on Carnot groups. Math. Ann. 325 (2003), 97-122.

[5] Bellaïche, A. And Risler, J.-J. (eds.): Sub-Riemannian Geometry. Progress in Mathematics 144. Birkhäuser, 1996.

[6] Crandall, M., Ishit, H., and Lions, P. L.: User's guide to viscosity solutions of second order partial differential equations. Bull. Amer. Math. Soc. (N.S.) 27 (1992), no. 1, 1-67.

[7] Danielli, D., Garofalo, N. and Nhieu, D.-M.: Notions of convexity in Carnot groups. Comm. Anal. Geom. 11 (2003), no. 2, 263-341.

[8] Danielli, D., Garofalo, N., Nhieu, D. and Tournier, F.: The Theorem of Busemann-Feller-Alexandrov in Carnot groups. Comm. Anal. Geom. 12 (2004), no. 4, 853-886.

[9] Folland, G. B., And Stein, E. M.: Hardy spaces on homogeneous groups. Mathematical Notes 28. Princeton University Press, 1982.

[10] Gutiérrez, C. and Montanari, A.: Maximum and comparison principles for convex functions on the Heisenberg group. Comm. Partial Differential Equations 29 (2004), no. 9-10, 1305-1334.

[11] Gutiérrez, C. and Montanari, A.: On the second order derivatives of convex functions on the Heisenberg group. Ann. Sc. Norm. Super. Pisa Cl. Sci. (5) 3 (2004), no. 2, 349-366.

[12] Jensen, R.: Uniqueness of Lipschitz extensions: minimizing the sup norm of the gradient. Arch. Rational Mech. Anal. 123 (1993), 51-74.

[13] Lu, G., Manfredi, J. J. and Stroffolini, B.: Convex functions on the Heisenberg Group. Calc. Var. Partial Differential Equations 19 (2004), no. $1,1-22$. 
[14] Magnani, V.: Lipschitz continuity, Aleksandrov theorem and characterizations for $H$-convex functions. Math. Ann. 334 (2006), no. 1, 199-233.

[15] Trudinger, N.S. And Wang, X.-J.: Hessian Measures II. Ann. of Math. (2) 150 (1999), no. 2, 579-604.

[16] Trudinger, N.S. And Wang, X.-J.: On the weak continuity of elliptic operators and applications to potential theory. Amer. J. Math. 124 (2002), $369-410$.

[17] Wang, C.: The Aronsson equation for absolute minimizers of $L^{\infty}$ functionals associated with vector fields satisfying Hörmander's condition. Trans. Amer. Math. Soc. 359 (2007), 91-113.

[18] Wang, C.: Viscosity convex functions on Carnot Groups. Proc. Amer. Math. Soc. 133 (2005), 1247-1253.

Recibido: 1 de marzo de 2005

Revisado: 6 de octubre de 2005

Petri Juutinen

Department of Mathematics and Statistics

P.O.Box 35

FIN-40014 University of Jyväskylä, Finland

peanju@math.jyu.fi

Guozhen Lu

Department of Mathematics

Wayne State University

Detroit, MI 48202, USA gzlu@math. wayne.edu

Juan J. Manfredi

Department of Mathematics

University of Pittsburgh

Pittsburgh, PA 15260, USA

manfredi@pitt.edu

Bianca Stroffolini

Dipartimento di Matematica e Applicazioni Università di Napoli Federico II

Via Cintia, 80126 Napoli, Italy

bstroffo@unina.it

First author supported by the Academy of Finland, project \#80566. Second author partially supported by NSF award DMS-0500853. Third author partially supported by NSF award DMS-0500983. Fourth author partially supported by COFIN02-MIUR, National Project "Calculus of Variations". 